during the past 13 years in Canada where, have great difficulty in affording to smoke? possibly because an actual fee for the surgery -I am, etc., is involved, the situation is more individualized and contractual, with the consequent possibility of litigation in the event of failure. Therefore for personal and legal reasons I could not guarantee sterility if any spermatozoa-motile or non-motile-were present in the semen. In the early years I required only one negative specimen but later increased this to two. Because of two pregnancies, with motile spermatozoa, I have for the past five years followed a routine of two negative specimens, two weeks apart, two months after the operation followed by a third negative specimen one month later. Thus the patient and $I$ are satisfied that every reasonable precaution has been taken. If a specimen shows any sperm, then specimens are repeated monthly for 8-12 months. At the end of this variable period a second operation is performed as before under local anaesthetic with no difficulty, using a fresh site on the vas if there is any granulomatous tissue formation from the previous surgery. A total of 14 patients, 12 with non-motile sperm and two with large motile sperm counts, had repeat vasectomies performed and in every case was aspermic one month after operation.

It was noted that, generally speaking, the older the patient, the longer the period required to attain azoospermia, the oldest (75 years) taking 18 months. While I have a routine based on a period of time, the vital factor is the frequency of coitus, which in the older patient with failing sexual function requires more time and more ejaculations to complete evacuation of the spermatozoa. The time schedule was strictly adhered to in order that if recanalization takes place, which is more probable in the first two months, it will show up in the routine tests. It would appear that sperm present in the semen after 50 intercourses indicates recanalization, and reoperation should be considered. Intermittant aspermia may be due to a temporary blockage of a recanalizing vas. No third vas was encountered and it is probable that its functional existence is to provide the surgeon with a reason to give the patient for failure to attain sterility.

It is unlikely and unethical that the question whether a patient with non-motile sperms in his specimen is capable of impregnationother specimens may contain motile spermatozoa-will be resolved as it is not in the interest of the patient, his wife, or the child to prove that the husband is not the father. If the doctor is not aware of this the patient and his wife are, and will refuse the confrontation and consequences.-I am, etc.,

Ardfern by Lochgilphead,

E. S. LIVINGSTONE Argyll

\section{Lung Cancer and Smoking}

SIR,-After the latest depressing news about the increase in cigarette smoking I consider it inevitable that eventually legislation will have to be introduced prohibiting the use of tobacco or limiting its use to registered addicts only.

Should not the B.M.A. start now and lobby the Government seriously to consider legislation, or at least increase the prices so much that children under 14 years would
Hemel Hempstead.

R. C. HOBBS lipids. ${ }^{6}$

The phenomenon to which Dr. F. M. Martins and his colleagues draw attention (8 September, p. 544) is interesting and deserves further study, but we cannot yet assume that those newborn babies with relatively high triglyceride levels in cord blood will be the individuals who show the better known primary type IV hyperlipoproteinaemia in middle age. Careful and prolonged follow-up is needed and it could yet turn out that the cause of "newborn type IV hyperlipoproteinaemia" is obstetrical (in the broadest sense of the word) rather than genetic. monstrate tubal patency during diagnostic laparascopy. The usual preparation is methylene blue injection, U.S.P., $1 \%$ in water in 10-ml ampoules (Harvey Laboratories, Inc., Philadelphia). The $\mathrm{pH}$ is 6.07. I wish to emphasize that this should be diluted liberally. Because the colour is very strong 1 $\mathrm{ml}$ diluted in $1000 \mathrm{ml}$ of sterile normal saline is sufficient to show clearly after spill into the peritoneal cavity.

An average injection would be $10 \mathrm{ml}$ and if given undiluted, straight from the ampoule, can provoke an acute reaction with signs of pelvic peritonitis lasting for some weeks, presumably following a "chemical salpingitis." There is also the problem of leakage into uterine blood vessels. The manufacturer's leaflet relating to intravenous administration of undiluted dye specifies the precaution of slow injection over minutes of a dose calculated on a body weight basis and mentions adverse reactions of nausea, abdominal and precordial pain, dizziness, headache, profuse sweating, mental confusion, and the formation of methaemoglobin.-I am etc.,

Department of Obstetrics and Gynaecology,

J. C. O’Sullivan Hammersmith Hospital,
London $W .12$

\section{Type IV Hyperlipidaemia in Cord Blood}

SIR,-Though Kwiterovitch et al., ${ }^{1}$ have recently reported triglyceride concentrations in cord blood averaging $37 \mathrm{mg} / 100 \mathrm{ml}$, with a standard deviation of only $15 \mathrm{mg}$, in 36 normal infants, evidence was published 11 years ago that a proportion of newborn babies have relative hypertriglyceridaemia. Brody and Carlson $^{2}$ measured triglycerides and other lipids in cord blood from 52 unselected newborn infants. The mean concentration of their plasma triglycerides was $0.38 \mathrm{mmol} / 1$. (approximately $33 \mathrm{mg} / 100 \mathrm{ml}$ ) but the frequency distribution was significantly skewed to the right-so that a standard deviation could not be calculated-and values ranged up to $0.85 \mathrm{mmol} / 1$. or $74 \mathrm{mg} / 100 \mathrm{ml}$. The upper tail of the distribution might, if one wished, be called relative type IV hyperlipidaemia in the newborn.

Brody and Carlson suggested that genetic factors could be responsible for this phenomenon. However, the last weeks of pregnancy and birth itself are a time of stress and metabolic changes whose intensity varies between individuals. Some degree of fatty liver appears to be quite common in the newborn. ${ }^{4}$ Pre- $\beta$-lipoprotein, which carries endogenous triglycerides in plasma, is the predominant lipoprotein in cord blood but its concentration falls off during the first few days of life, while that of $\beta$-lipoprotein increases. ${ }^{5}$ There are accompanying major
Department of Nutrition,

A. S. TRUSWELI

London $\mathbb{W} .8$

1 Kwiterovitch, P. O., jun., Levey, R. I., and Fredrickson, D. S., Lancet, 1973, 1, 118 . Acta, 1962, 7, 694 .

Dorkin, J. R., and Weinberg, T., Archives of Pathology, 1949, 48, 578

Benitez, M. R. E., Archives of Pathology, 1952, 54, 378.

Friis-Hansen, B. and Clausen, J., Zeitschrift fur Ernährungswissen-schaft, 1971, 10, 253.

6 Clausen, J., and Friis-Hansen, B., Zeitschrift fur Ernährungswissen-schaft, 1971, 10, 264.

\section{Drug-induced Platelet Antibodies}

SIR,-We read with interest the letter of Drs. C. Davidson and S. M. Manohitharajah (8 September, p. 545), who reported the presence of specific platelet antibodies in a patient who developed thrombocytopenia while receiving phenylbutazone therapy. With the increasing incidence of druginduced blood dyscrasias it would appear to be more and more relevant to search for mediating antibodies and we would like to report another illustrative case.

A 77-year-old woman presented with a 24-hour history of epistaxis and rectal bleeding, with spontaneous bruising for two weeks. She had been taking phenylbutazone regularly for 12 years to alleviate arthritic pain and for three years she had been taking quinine sulphate as treatment for nocturnal cramps. We were unable to ascertain any recent alteration in dosage or proprietary brand. Examination revealed a pale woman with generalized bruising and purpura over the limbs and trunk. Petechial haemorrhages in the mouth and a flame haemorrhage in the left fundus were noted, together with evidence of recent epistaxis and rectal findings. leucocyte count $1,200 / \mathrm{mm}^{3}$, and platelet count $10,000 / \mathrm{mm}^{3}$. Bone marrow aspiration revealed a mildly hypocellular marrow with macronormoblastic erythropoiesis and delayed maturation of granulopoiesis. Megakaryocytes showed reduced platelet budding. There was no evidence of bone marrow infiltration. Tests for antinuclear factor and L.E. cells were negative, as were Ham's test and screening for urinary haemosiderin. Serum vitamin $B_{12}$ and folate levels were normal. The Direct Coombs test was negative. Tests for leucocyte and platelet agglutinins were also negative, but a complement fixation test was strongly positive for incomplete platelet antibodies in the presence of quinine sulphate. This was negative in the presence of phenylbutazone.

All drugs were withheld and, apart from reverse barrier nursing, no active therapy was given. After eight days her haemoglobin level had risen to $10.3 \mathrm{~g} / 100 \mathrm{ml}$, total leucocyte count to $4,600 / \mathrm{mm}^{3}$, $10.3 \mathrm{~g} / 100 \mathrm{ml}$, total leucocyte count

It would appear that the pancytopenia resulted from the combined effects of phenylbleeding. There were no other abnormal clinical

Her haemoglobin level was $9.7 \mathrm{~g} / 100 \mathrm{ml}$, total 
butazone and quinine sulphate. The induction of platelet antibodies by quinine has been described ${ }^{2}$ and the mechanism of development is probably identical with that caused by apronal (Sedormid). The effect of phenylbutazone as a generalized bone marrow suppressor is well known and it is of interest that phenylbutazone-induced platelet antibodies have been reported previously. ${ }^{4}$

The precise mechanisms involved in our case are open to some speculation, though it is known that phenylbutazone in combination with other drugs produces pancytopenia more commonly than when given alone. ${ }^{5}$ It seems likely that bone marrow suppression by the combination of phenylbutazone and quinine sulphate was the major factor in the pathogensis of the pancytopenia, but the platelet antibodies must be considered to have been a contributory factor in producing such a severe degree of thrombocytopenia.

Evidence accumulates that the drug induction of antibody-mediated thrombocytopenia is not so rare as has been believed; in common with Drs. Davidson and Manohitharajah we believe that there is good reason to look for platelet antibodies in thrombocytopenias thought to be druginduced, as their presence provides a rationale for effective therapy and a guide for subsequent reintroduction of the offending drug.

We thank Dr. K. Goldsmith of the M.R.C. Blood Groups Reference Laboratory for performing the tests for leucocyte and platelet antibodies and Professor A. Guz for permission to report his patient.

-We are, etc.,

A. C. KEAT

R. G. RAWBONE

J. MITRA

T. R. MITCHEL

Departments of Haematology and Medicine,

Charing Cross Hospital,
London W.6

1 Miescher, P. A., Seminars in Hematology, 1973, 2 10.179.

3 Helmly, R. B., Bergin, J. J., and Shulman, N. R. Archives of Internal Medicine, 1967, $120,59$. - Meiers, H. G., Gehrmann, G., and Gahlen, W. Deutsche medizinische Wochenschrift, 1963, 88, 580 .

- Erslev, A. J., and Wintrobe, M. M., foumal of the American Medical Association, 1962, 181 114.

"Thou shalt not kill; but . . ."

SIR,-Mr. G. Keys Smith and Mr. E. Durham Smith (27 October, p. 189) are certainly not the first and probably not the last to take Clough's lines "Thou shalt not kill; but need'st not strive Officiously to keep alive" out of context to support an argument. The poem from which the lines are taken, "The Latest Decalogue," is satirical, juxtaposing in each couplet a commandment and a wrong reason for obeying it. This is obvious from the lines following those which are so commonly quoted:

"Do not adultery commit

Advantage rarely comes of it:

Thou shalt not steal; an empty feat,

When it's so lucrative to cheat."

-I am, etc.

Marton, Middlesbrough

\section{Calcification in Burkitt's Lymphoma}

SIR,-It is the purpose of this letter to document what appear to be the first cases of Burkitt's lymphoma with calcification in abdominal tumour masses before treatment.

Over 350 cases of Burkitt's lymphoma have been reported from Ibadan, where this tumour accounts for over $50 \%$ of all childhood malignancies. Abdominal involvement is present in $30-70 \%$ of cases reported from various parts of the world. In the abdomen multiple intraperitoneal and retroperitoneal sites are usually involved, commonly the kidneys, pancreas, adrenals, ovaries, gastrointestinal tract, and mesenteric and retroperitoneal lymph nodes. I have been unable to find any reported case of Burkitt's lymphoma with radiographically visible calcification within abdominal tumour masses prior to treatment. A Medlars computer search of the literature from January 1970 to May 1973 was unable to locate any citations for this entity and Dr. D. P. Burkitt is also unaware of any such case (personal communication, 1973). Whittaker ${ }^{12}$ reported cases from Kenya in which calcification developed after treatment.

Case 1.-A 7-year-old Nigerian boy presented with a brief history of abdominal distension and pain. He had hepatomegaly and splenomegaly, and multiple masses were palpable in his fluid-filled abdomen. An intravenous pyelogram showed essentially normal renal collecting systems with some delayed drainage on the left. A cystic calcification was present in front of and slightly below the left kidney (see figs). Cytological examination of abdominal fluid showed typical Burkitt lymphoma cells. He had a poor response to chemotherapy and died about one month later. At necropsy there was a large mass in the abdomen, primarily on the left, infiltrating the spleen, left kidney, pancreas, para-aortic lymph nodes, omentum, and the wall of the colon. There were also nodular deposits in the spleen. Histological examination confirmed the diagnosis of Burkitt's lymphoma. Unfortunately the specific site of calcification within the tissue/tumour masses was not identified histologically at necropsy and tissue was not available for re-study at a later date.

Case 2.-A 12-year-old boy with abdominal swelling and a mass on the right showed deformity of the renal collecting system and ureter on the right. Bilateral calcifications were present posteriorly in a suprarenal location. Biopsy of the right kidney showed the histological and cytological appearances of Burkitt's lymphoma. The patient was then lost to follow-up.

-I am, etc.

Department of Radiology,

University of Ibadan,

Ibadan, Nigeria

1 Whittaker, L. R., Australasian Radiology, 1970,

14, 197.
Whittaker, L. R., Clinical Radiology, 1973, 24, 339.

\section{Pyridoxine and Parkinsonism}

SIR,-Dr. A. B. Carter (27 October, p. 236) ڤึ points out that pyridoxine-containing com- $\vec{\circ}$ pounds are contraindicated in the treatment of nausea in patients who are receiving $\vec{\omega}$ levodopa for Parkinsonism. Dr. Carter states $\$$ that he usually prescribes metoclopramide but fails to mention that this compound itself may cause Parkinsonism ${ }^{1}$ which will not be relieved by increasing the dose of levodopa. $\infty$ -I am, etc.

Nevill Hall Hospital,

SHARON M. CARTER Abergavenny, Mon

1 Laurence, D. R, Clinical Pharmacology, 4th edn, 0 Edinburgh, Churchill Livingstone, 1973.

\section{Ideal Diet}

SIR,-There has been recent correspondence

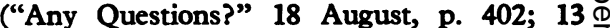
October, p. 103) as to an ideal diet which $\vec{A}$ will not elevate blood cholesterol. It was suggested that a carbohydrate-restricted diet, $\omega$ allowing freedom to eat "non-carbohydrate" foods, including meat, eggs, and dairy products, is suitable. These latter foods are high in saturated fats and cholesterol, which may cause elevation of the blood cholesterol. $\frac{\circ}{\mathbb{D}}$ However, the claim is made that on such a $\bigcirc$ diet the amounts of fat and protein are self- $\overrightarrow{\vec{B}}$ limiting when carbohydrate intake remains 3 around $60 \mathrm{~g}$ a day.

A detailed study ${ }^{1}$ has recently been undertaken in which precisely this dietary advice (described in the paper as the "Yudkin diet") was compared with a more usual calorierestricting diet. On the low-carbohydrate diet $\overline{0}$

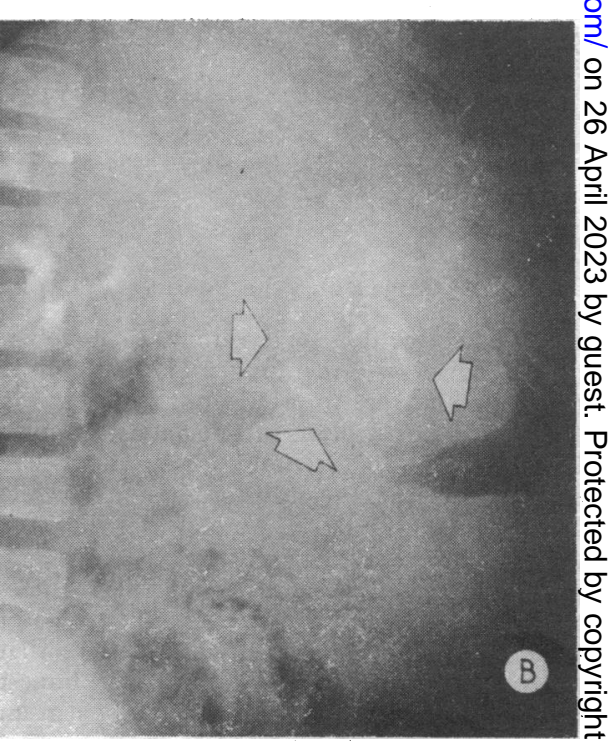

Fig. A: Abdominal radiograph 15 minutes after intravenous injection of pyelographic contrast material. Fig. B: lateral view.

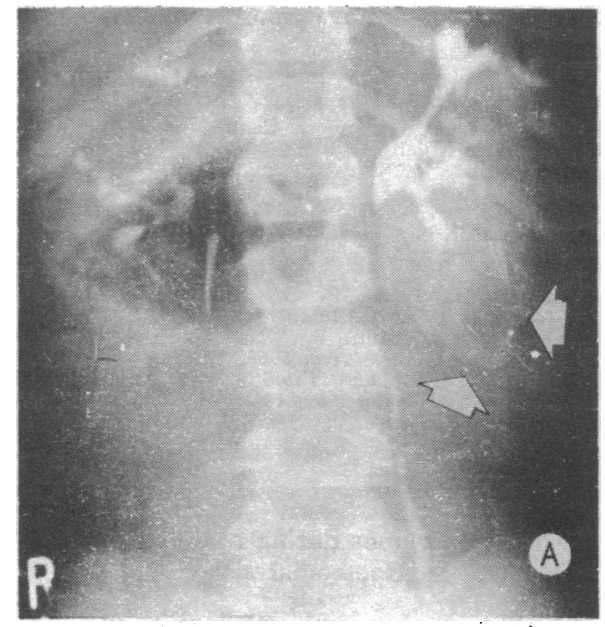

.

.

(1)

